\title{
Hurricane Kinetic Energy Spectra from In Situ Aircraft Observations
}

\author{
P. TRENT Vonich AND GREgORY J. HAKIM \\ Department of Atmospheric Sciences, University of Washington, Seattle, Washington
}

(Manuscript received 12 September 2017, in final form 22 April 2018)

\begin{abstract}
Since the pioneering paper by Nastrom and Gage on aircraft-derived power spectra, significant progress has been made in understanding the wavenumber distribution of energy in Earth's atmosphere and its implications for the intrinsic limits of weather forecasting. Improvements in tropical cyclone intensity predictions have lagged those of global weather forecasting, and limited intrinsic predictability may be partially responsible. In this study, we construct power spectra from aircraft data of over 1200 missions carried out by the National Oceanic and Atmospheric Administration (NOAA) and Air Force Reserve Command (AFRC) Hurricane Hunters. Each mission is parsed into distinct flight legs, and legs meeting a specified set of criteria are used for spectral analysis. Here, we produce power spectra composites for each category of the SaffirSimpson scale, revealing a systematic relationship between spectral slope and storm intensity. Specifically, as storm intensity increases, we find that 1) spectral slope becomes steeper across scales from 10 to $160 \mathrm{~km}$ and 2) the transition zone where spectral slope begins to steepen shifts downscale.
\end{abstract}

\section{Introduction}

The challenge of enhancing hurricane wind speed forecasts is thought to involve several aspects of the modeling process. Rogers et al. (2013) succinctly summarized the issues in their aircraft-based hurricane investigation, stating that the hurricane intensity skill shortfall exists within microphysical processes and predictability constraints. Recent research connected with the Hurricane Aerosol and Microphysics Program (HAMP) - a sister project to the Hurricane Forecast Improvement Project (HFIP) - supports the importance of microphysical processes to intensity forecasting (Rosenfeld et al. 2007; Khain et al. 2010; Rosenfeld et al. 2011). The fundamental forecasting limitations imposed by hurricane predictability are not fully known, especially from an observational perspective, which motivates this paper on aircraft-based power spectra.

The study of atmospheric predictability revolves around the growth of errors. Given the power spectrum of a fluid flow, conclusions can be drawn as to how quickly small-scale errors will contaminate large scales. Lorenz (1969) was the first to demonstrate this relationship, spurring research into the field of atmospheric predictability that continues today. He demonstrated that systems governed by a $k^{-p}$ power law have limited

Corresponding author: P. Trent Vonich, tvonich@gmail.com predictability when $p<3$, whereas systems governed by $p \geq 3$ have unlimited predictability. While Lorenz (1969) considered the predictability of flows within the two-dimensional vorticity equation framework, Rotunno and Snyder (2008) applied Lorenz's spectral approach to the surface quasigeostrophic equations and confirmed that the structure of the energy spectrum strongly influences predictability.

Observational documentation of large-scale atmospheric spectra followed from the 1975-79 NASA-led Global Atmospheric Sampling Program. During this program, Boeing 747s fitted with special instrumentation gathered atmospheric data along commercial flight paths, providing Nastrom and Gage (1985) with the data to complete their seminal paper on wind and temperature power spectra. Their study yielded a power spectrum with a $k^{-5 / 3}$ power law for wavelengths less than $400 \mathrm{~km}$ and a $k^{-3}$ power law for wavelengths larger than $400 \mathrm{~km}$. These findings dispelled theories that-at the time-proposed a mesoscale energy gap. The continuous increase in energy from small to large scales demonstrated by Nastrom and Gage (1985) corroborated the findings of Lilly and Petersen (1983) and are consistent with Vinnichenko (1970), Balsley and Carter (1982), and Chen and Wiin-Nielsen (1978). While Nastrom and Gage (1985) provide an observational foundation for spectral properties of the extratropical upper troposphere, the spectral properties of specific atmospheric 
features, such as hurricanes, remains an open question that we explore here. We note that the link between properties of homogeneous and isotropic turbulence and real atmospheric flows is complicated by inhomogeneities, such as ubiquitous fronts and jet streams for the extratropical spectrum and the primarily axisymmetric circulation of hurricane vortices. Nevertheless, documenting observed spectra provides a basis for comparison with numerical simulations (Skamarock 2004) and a source for theories that seek to explain observed phenomena.

The goal of this paper is to document the observed spectrum of hurricanes using in situ aircraft observations. Just as Nastrom and Gage (1985) created a climatology of wavenumber spectra for the upper troposphere, we present a climatology of wavenumber spectra for hurricanes as a function of the Saffir-Simpson scale. The remainder of the paper is structured as follows: In section 2, we describe how the in situ aircraft observations are taken and then discuss our methods for generating hurricane spectra in section 3. Results are presented in section 4, and we summarize our findings and ideas for future work in section 5 .

\section{Data}

\section{a. AFRC and NOAA Hurricane Hunters}

Every hurricane season, the National Oceanic and Atmospheric Administration (NOAA) Hurricane Hunters fly WP-3D aircraft from MacDill Air Force Base (AFB), Florida, to conduct atmospheric reconnaissance missions in the Atlantic basin and eastern North Pacific. The U.S. Air Force Reserve Command (AFRC) operates their own hurricane-hunting unit, the 53rd Weather Reconnaissance Squadron (53WRS), flying WC-130s out of Keesler AFB, Mississippi. Aircraft-mounted instruments record wind speed $\left[ \pm 4 \mathrm{knots}\left(\mathrm{kt} ; 1 \mathrm{kt}=0.51 \mathrm{~m} \mathrm{~s}^{-1}\right)\right]$, wind direction $\left( \pm 10^{\circ}\right)$, GPS position $[ \pm 3$ nautical miles $(\mathrm{n} \mathrm{mi}$; $1 \mathrm{n} \mathrm{mi}=1.85 \mathrm{~km})$ ], temperature, dewpoint, mixing ratio, altitude, and other variables (Federal Coordinator for Meteorological Services and Supporting Research 2017).

Missions analyzed in this study are separated into three datasets: 1) NOAA 1-s data, 2) AFRC 1-s data, and 3) AFRC 10-s data. For flights occurring from 1960 to 2009, AFRC wind data are available at 10 -s intervals, where each wind value represents a trailing average of the wind speed (53rd Weather Reconnaissance Squadron 2016). After 2009, the 53WRS upgraded to 1-s data output; however, each wind value in the AFRC 1-s dataset represents a 30 -s average of the wind speed taking place during the $15 \mathrm{~s}$ before and after a specified time (53rd Weather Reconnaissance Squadron 2016). NOAA wind
TABLE 1. Collection of mission statistics and power spectra results organized by storm intensity.

\begin{tabular}{cccccc}
\hline \hline Category & Missions & $\begin{array}{c}\text { Flight } \\
\text { legs }\end{array}$ & $\begin{array}{c}\text { Steepest } \\
\text { slope }\end{array}$ & $\begin{array}{c}\text { Bin of steepest } \\
\text { slope }(\mathrm{km})\end{array}$ & $\begin{array}{c}\text { Mean } \\
\text { slope }\end{array}$ \\
\hline 5 & 43 & 274 & -3.1 & $43-68$ & -2.2 \\
4 & 120 & 760 & -2.4 & $43-68$ & -2.0 \\
3 & 114 & 718 & -2.5 & $68-106$ & -2.0 \\
2 & 146 & 922 & -2.3 & $106-166$ & -1.8 \\
1 & 274 & 1597 & -2.1 & $68-106$ & -1.8 \\
TS & 583 & 2973 & -1.9 & $43-68$ & -1.7 \\
\hline
\end{tabular}

speed data are output at $1 \mathrm{~s}$ as well and represent the average wind speed during a given second, where wind speeds are measured at a rate faster than $1 \mathrm{~Hz}$. Both NOAA and AFRC wind data are adjusted for aircraft angle of attack and sideslip angle. Wind speeds are recorded at the nearest knot for the AFRC and the nearest tenth of a meter per second for NOAA. Additional detail regarding data processing, data quality, and flight procedures can be found in the appendix.

For this study, we analyze data from tropical storms (TSs) and hurricanes from 1980 to 2015, beginning with Hurricane Allen and ending with Hurricane Patricia. After screening all available missions for quality and relevance (further explanation in section 3a), 1280 missions were found suitable for analysis. These 1280 missions are spread across 217 named storms, and a breakdown of their intensities is given in Table 1. Older missions-particularly those from the 53WRS in the 1980s and 1990s - do not include aircraft speed data. Selection of suitable missions and calculation of power spectra in the absence of aircraft speed are addressed in section $3 \mathrm{c}$.

\section{b. NOAA best track data}

To ensure a consistent sample, we require that flight legs pass through the radius of maximum wind (RMW) to be considered for spectral analysis. Passing through the RMW is useful for our analysis because this region of the hurricane has the greatest inertial stability. Strong inertial stability makes the dynamics more barotropic, potentially steepening power spectra and influencing predictability. To determine if a flight leg has intersected the RMW, NOAA best track (NBT) data were employed (detailed explanation in section 3b). Maximum 1-min surface wind speed, minimum central pressure, and storm position for North Atlantic and eastern Pacific storms are logged every $6 \mathrm{~h}$ by the National Hurricane Center. The Joint Typhoon Warning Center accomplishes the same task for the western Pacific, South Pacific, southern Indian, and northern Indian. Occasionally the AFRC Hurricane Hunters travel to the western Pacific during the North Atlantic's dormant winter months, but the vast majority 
( $>99 \%$ ) of storms evaluated during this study are from the North Atlantic and eastern Pacific.

\section{Methods}

\section{a. Selecting suitable missions}

In this study, 1280 missions are considered. To distill all available missions into a set suitable for analysis, a subjective screening method is applied. Mission GPS data are visually compared to the NBT position during the time of hurricane intercept. If the mission's flight pattern coincides with the storm position from the NBT data, then the storm is retained for further evaluation. Plotting the flight tracks in this way easily exposes ferry, ocean wind survey, and synoptic surveillance flights. These missions do not involve hurricane intercepts, so they are not analyzed. Missions are classified on the Saffir-Simpson scale based on the maximum sustained winds of the NBT data point closest to the intercept time. Given that strong inertial stability is fundamental to our hypotheses about hurricane power spectra (hypotheses that will be evaluated in future studies), missions from tropical depressions are not included in the analysis. If NBT data are not available within $3 \mathrm{~h}$ of a hurricane intercept, the mission is not analyzed.

\section{b. Identifying flight legs}

Since we are only concerned with power spectra of flights intersecting the RMW, we determine which flight legs meet this constraint. A mission's flight track is separated into legs (Fig. 1a). Hurricane intercept times do not correspond exactly to NBT records, so storm position is linearly interpolated between the two closest NBT position points based on the time of intercept. If a flight leg passes within $55 \mathrm{~km}$ of a storm's center, it is considered to have penetrated the RMW (Fig. 1b). ${ }^{1}$ An algorithm is then applied to the legs meeting the $55-\mathrm{km}$ criterion, and an exact RMW location is determined. Flight legs shorter than $10 \mathrm{~km}$ are not analyzed. This is due to wind speed variability, which makes it difficult to identify the RMW over short distances. Finally, if a flight leg extends across the full diameter of the storm, two new flight legs are created-one on each side of the RMW (see a hypothetical example in Fig. 2 and a realworld example in Fig. 3).

\section{c. Spectral analysis}

Wind speeds are linearly interpolated to a regular grid for each flight leg. Grid spacing for a flight leg is defined as the

\footnotetext{
${ }^{1}$ The average RMW for cat-2, -3 , and -4 storms was found by Hsu and Yan (1998) to be $48 \mathrm{~km}$. Increasing this distance to the $55-\mathrm{km}$ radius $-0.5^{\circ}$ of latitude - allows for a small buffer given a storm's continual movement during a mission.
}

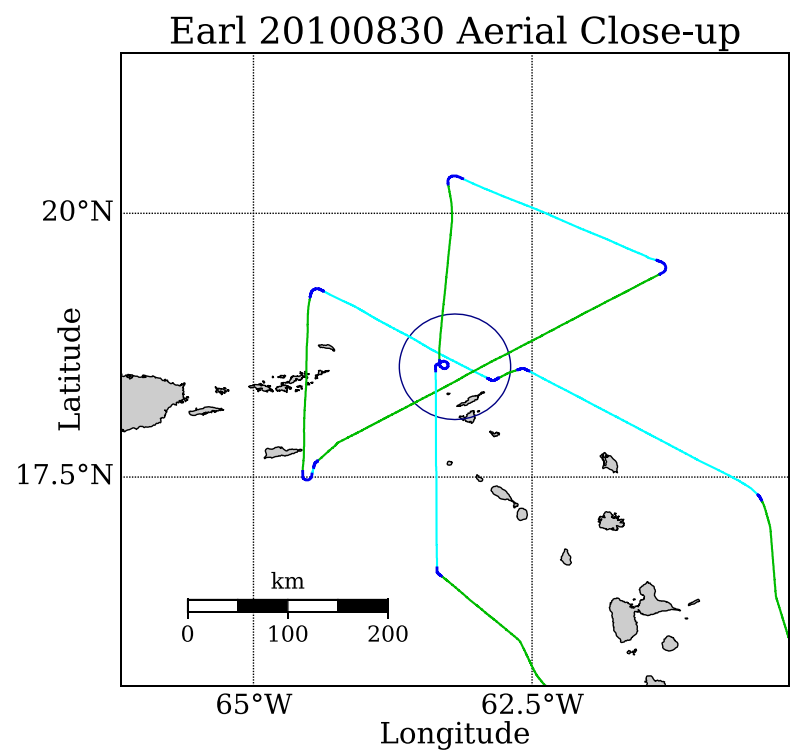

(a)

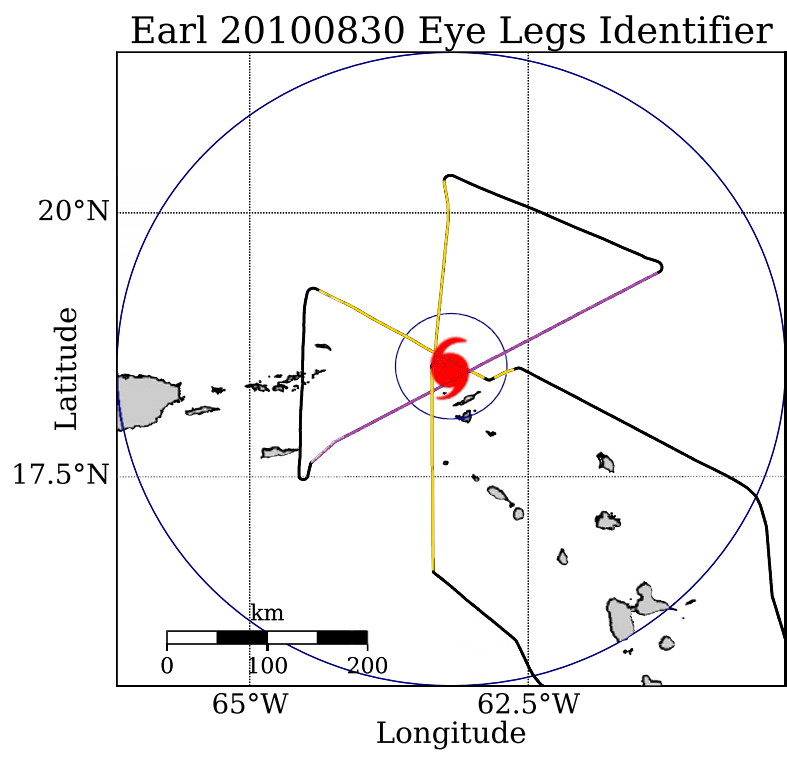

(b)

FIG. 1. (a) Distinct flight legs are shown as light blue and green. An algorithm partitions the overall flight path into unique legs when a ground track variance threshold is exceeded, indicating a turn (dark blue). (b) Flight legs intersecting the $0.25^{\circ}$-radius inner circle are highlighted in orange, with the purple leg corresponding to the velocity profile shown in Fig. 3 .

plane's average airspeed multiplied by the sampling interval (1 or $10 \mathrm{~s}$ ). Nastrom and Gage (1985) operate under the assumption that Boeing 747 ground speeds (roughly $250 \mathrm{~m} \mathrm{~s}^{-1}$ ) are large compared to the speed of relevant atmospheric disturbances - that is to say, disturbances move 


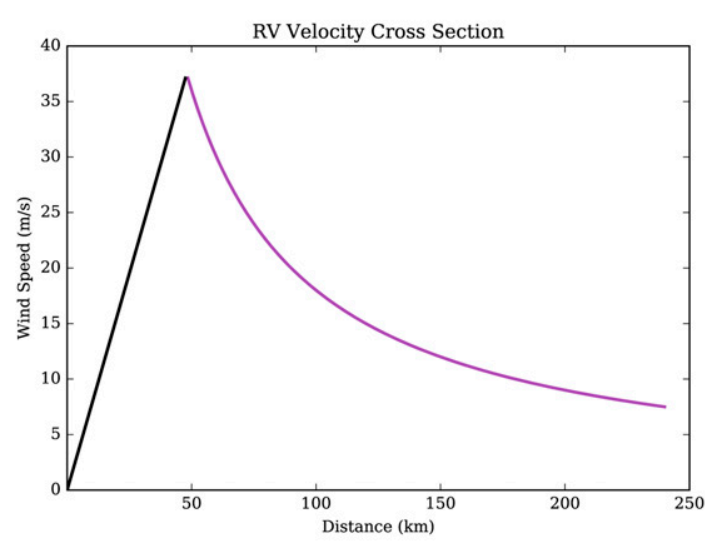

(a)

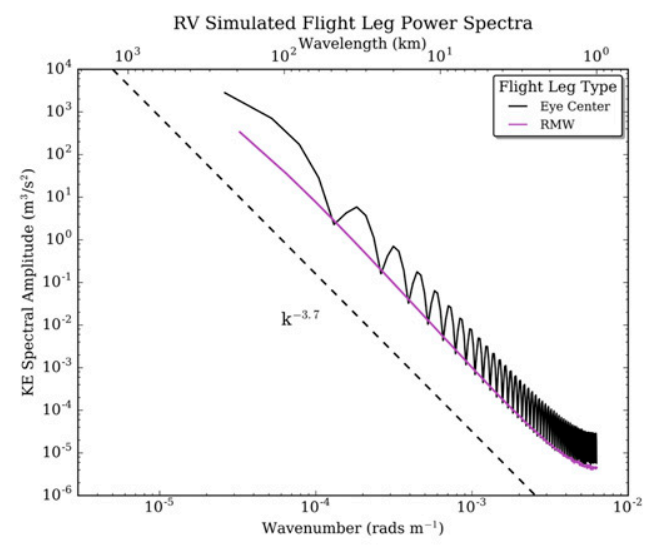

(b)

FIG. 2. (a) A Rankine vortex radial velocity profile is shown with an RMW located at $48 \mathrm{~km}$. The purple curve represents winds following RMW truncation, and the black curve shows the truncated portion of a hypothetical flight leg. (b) The power spectra of the retained wind data (purple) and the retained wind data plus the truncated information (black). Aliasing occurs in the black curve because the sharp discontinuity is not removed. The purple curve has a log slope of approximately -3.7 .

negligible distances during the time it takes to sample them. As a result, Nastrom and Gage (1985) simply prescribe wind values to a $250-\mathrm{m}$ grid. Lilly and Petersen (1983) attempt to address the subtleties of relative motion between aircraft and disturbances by using mean airspeed $\left(240 \mathrm{~m} \mathrm{~s}^{-1}\right)$. Understanding that no all-encompassing method could account for every nuance regarding relative motion, Lilly and Petersen conclude most resulting errors cancel out in the overall mean. Given the symmetric flight paths conducted by the 53WRS and NOAA, we draw the same conclusion and assume that any errors caused by relative motion cancel out in the average over a large sample. Moreover, hurricane winds are almost entirely azimuthal at aircraft flight levels. Consequently, ground speed and airspeed are virtually the same. As a result, we simply use ground speed to calculate the normalizing factor explained in the next paragraph. ${ }^{2}$

After interpolating wind speeds to a regular grid, the mean and endpoint-to-endpoint linear trend are removed from each leg. A fast Fourier transform (FFT) is applied, and the squared Fourier coefficients are used to produce a periodogram for each flight leg (Fig. 4). Since each flight leg has a unique grid spacing and average speed, the spectral amplitudes of each periodogram are multiplied by the normalizing factor, $\Delta x /(\pi N)$, where $\Delta x$ is the distance between grid points and $N$ is the number of data points in a flight leg (Durran et al. 2017). After normalization, the spectral amplitudes in each periodogram are

\footnotetext{
${ }^{2}$ AFRC 1-s missions occurring before 2005 (about $0.83 \%$ of all missions) do not contain ground speed or airspeed, so average ground speed is determined by flight leg length divided by time.
}

distributed across 17 logarithmically spaced wavenumber bins in the same manner as Lilly and Petersen (1983), and the points within each bin are averaged to create a power spectrum composite for each category of the Saffir-Simpson scale. Four spectral composites are produced: a NOAA composite, an AFRC 1-s composite, an AFRC 10-s composite, and a joint composite composed of all three datasets.

The maximum (minimum) wavenumber (wavelength) bin edge is $1 \times 10^{-2}, 2 \times 10^{-3}$, and $2 \times 10^{-3} \mathrm{rad} \mathrm{m}^{-1}$ for the NOAA 1-s, AFRC 1-s, and AFRC 10-s composites, respectively. Upper (lower) wavenumber (wavelength) bounds are set for the NOAA and AFRC 1-s datasets so as to discard wavenumbers affected by the moving

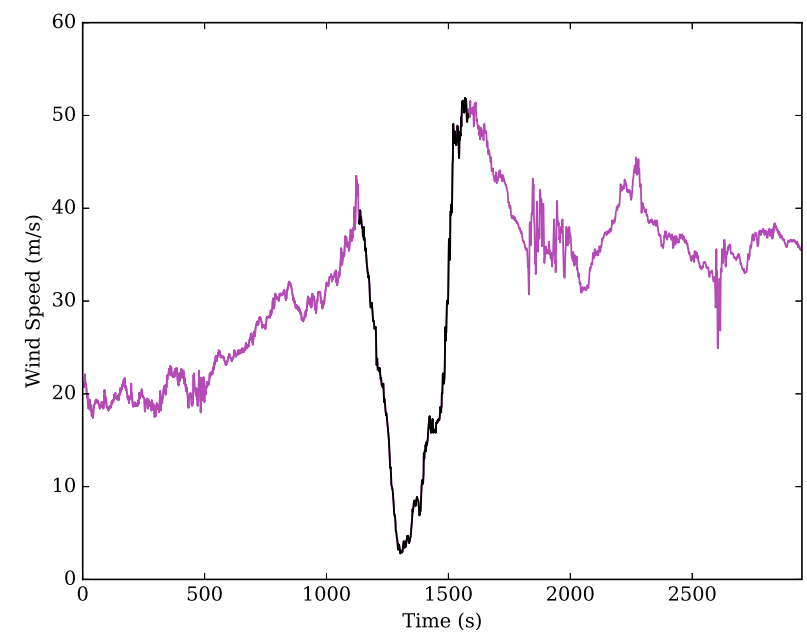

FIG. 3. Wind data from a flight leg spanning the full diameter of Hurricane Earl on 30 Aug 2010 are truncated at the RMW similar to Fig. 2a. The complete flight leg is separated into two smaller flight legs (purple), while the sharp discontinuities and eye (black) are removed. 


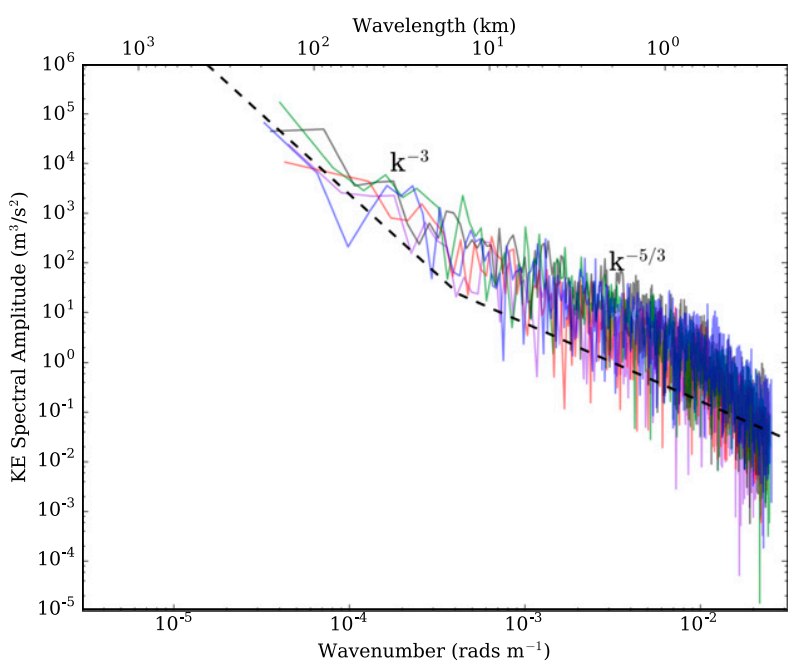

FIG. 4. Periodograms corresponding to flight legs from the mission shown in Fig. 1b; 7244 total periodograms (flight legs) are averaged to form the composite spectral profiles for each category of the Saffir-Simpson scale shown in Fig. 8. Dashed reference lines represent $k^{-5 / 3}$ and $k^{-3}$ slopes.

averages mentioned in section 2a. Though the AFRC 10-s data have no aliasing from averaging, $2 \times 10^{-3} \mathrm{rad} \mathrm{m}^{-1}$ is chosen as the upper wavenumber bound in order to remove scales aliased at the Nyquist frequency. Finally, the combined dataset has an upper wavenumber bound of $1 \times 10^{-2}$ $\operatorname{rad~m}^{-1}(3.1 \mathrm{~km})$ by default due to the bound applied to NOAA and a lower bound of $6.28 \times 10^{-6} \mathrm{rad} \mathrm{m}^{-1}$ $(1000 \mathrm{~km})$ to remove highly variable spectra caused by the small number of flight legs greater than $1000 \mathrm{~km}$. The NOAA, AFRC 1-s, and AFRC 10-s composites have no lower wavenumber bound.

Since hurricanes are far from homogeneous and isotropic, we expect at the outset that observed spectra may not sample isotopic homogeneous turbulence. As such, we might anticipate a contribution of the primary radial structure to the hurricane power spectrum. Similarly, one might also expect similar issues associated with spectra for the extratropical upper troposphere where long-haul aircraft flights primarily sample the structure of the westerly jet streams. Although we are not aware of null spectra posed in the literature on spectra for extratropical jets, here we present a null hypothesis for a contribution to the spectrum we might expect to find from sampling the dominant radial structure of hurricanes. A Rankine vortex $(\mathrm{RV})$ is a canonical vortex profile that serves this purpose. Treating the vortex as we do the flight-level data produces a steep spectrum (Fig. 2b). Here, the RV RMW is set to $48 \mathrm{~km}$. Shifting the RMW will change the kurtosis of the RV, but impacts on spectral slope are negligible for a reasonable real-world range $(24-72 \mathrm{~km})$. We observe oscillatory behavior in the spectrum (black line),

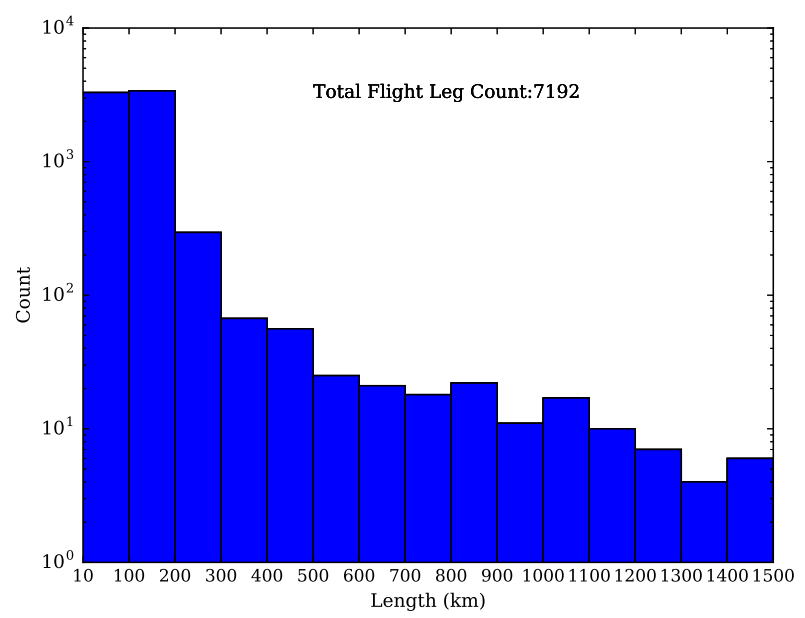

FIG. 5. Flight leg length distribution.

which is due to the discontinuity at the RMW. Analyzing information that excludes this discontinuity reveals a smooth spectrum (purple line) with a -3.7 slope. Having this background understanding for the RV spectrum, we also consider spectra from aircraft flight legs with and without truncation at the RMW, which allows us to assess the impact of this sharp feature on the spectra. Details regarding the method for this aspect are described below.

\section{Results}

\section{a. Power spectra composites}

From a total of 1280 missions, 7244 flight legs are analyzed; 7148 of these flight legs are $1000 \mathrm{~km}$ in length or less, and 96 are greater than $1000 \mathrm{~km}$ (Fig. 5). Leg truncation at the RMW tends to decrease the number of long flight legs, while increasing the number of short flight legs. Typical hurricane dimensions also tend to force the distribution away from the synoptic scales. The average storm latitude is $23.4^{\circ} \mathrm{N}$, with $97 \%$ of missions taking place in storms located between $10^{\circ} \mathrm{N}$ and $35^{\circ} \mathrm{N}$. Like Lilly and Petersen (1983), the standard deviation of each bin population is about the same magnitude as the bin's mean value or a bit larger for the lowest wavenumbers. A composite is presented for a reference dataset in which winds are less than $17.5 \mathrm{~m} \mathrm{~s}^{-1}$ (Fig. 6), for all three datasets combined (with and without RMW truncation; Fig. 7 and Fig. 8), and for each individual dataset (Fig. 9).

First, to establish a baseline from which to evaluate intensity-averaged spectra, a reference spectrum is produced using flight legs with less than tropical storm force winds, or $17.5 \mathrm{~m} \mathrm{~s}^{-1}$ (Fig. 6). These legs are from the same 1280 missions used to produce the intensity-averaged composites and primarily sample the tropical atmosphere inbound to and outbound from target storms. Such legs 


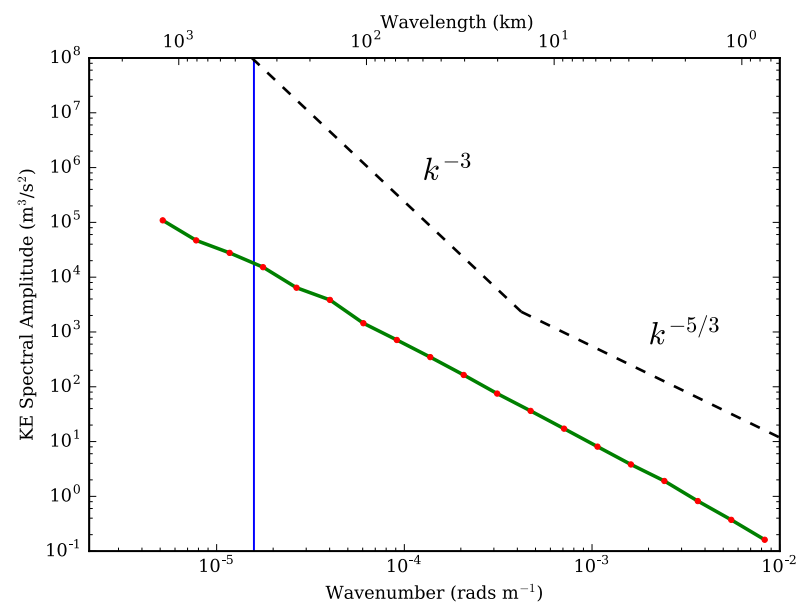

FIG. 6. Spectral composite of all flight legs containing wind speeds less than $17.5 \mathrm{~m} \mathrm{~s}^{-1}$.

are relatively uncommon since crews do not necessarily record data until a hurricane intercept has begun; 607 flight legs (120 legs greater than $400 \mathrm{~km}$ ) are utilized in Fig. 6, where we note an approximate $k^{-5 / 3}$ slope across all wavelengths with slight deviations at scales above $100 \mathrm{~km}$. The small sample size available to create Fig. 6 may partly explain why the slope does not steepen to $k^{-3}$ after $400 \mathrm{~km}$ as seen in Nastrom and Gage (1985). Nevertheless, we also see a slope shallower than $k^{-3}$ for all intensities in Fig. 8 - even scales larger than $400 \mathrm{~km}$. This suggests that the shallower spectral slope at these scales are generic to the tropical lower troposphere. The relatively weaker Coriolis force in the tropics compared to the midlatitude regions sampled by Nastrom and Gage (1985) may also partially explain the differences in slope results, but deeper insight into this question requires exploration in future research.

Next, we present the composite for all three datasets where flight legs are not truncated at the RMW (Fig. 7). For tropical storms, the spectrum is close to $k^{-5 / 3}$ for wavelengths shorter than about $20 \mathrm{~km}$ and steepens slightly toward longer wavelengths before becoming shallower than $k^{-5 / 3}$ for wavelengths greater than about $200 \mathrm{~km}$. As storm intensity increases, power increases preferentially for wavelengths of about $10-200 \mathrm{~km}$. Consequently, the spectral slope also increases in this range, reaching approximately $k^{-3}$ for category (cat)-3-5 storms, and decreases at longer wavelengths. At scales below about $10 \mathrm{~km}$, the spectrum remains close to $k^{-5 / 3}$ even as power increases across all scales as a function of storm strength.

Composites composed of flight legs that have been truncated at the RMW reveal spectral slopes that are shallower than those that include the RMW (Fig. 9). The shallower slopes are a result of removing the sharp wind field discontinuity experienced when proceeding from

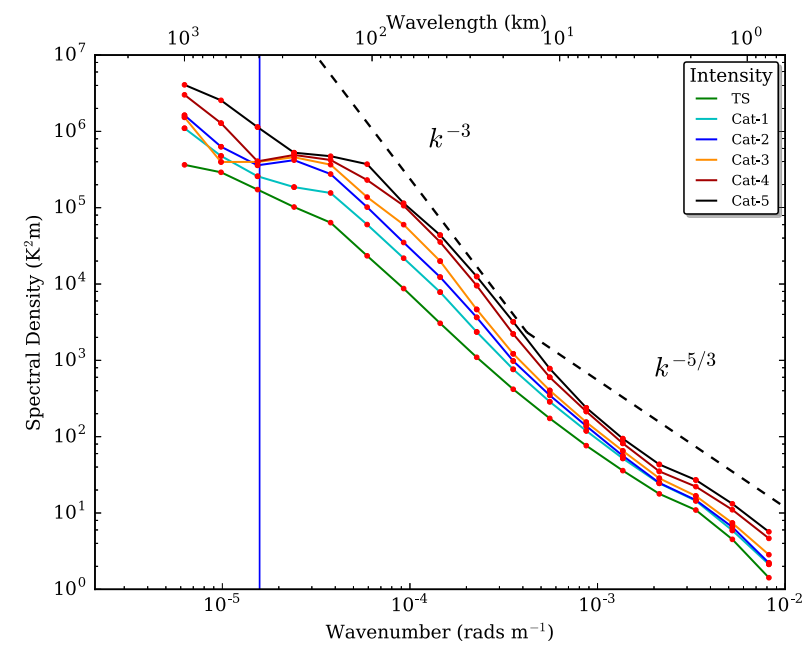

FIG. 7. Spectral composites of all three datasets. Flight legs are not truncated at RMW.

the RMW into the center of the eye. The decrease in slope steepness compared to Fig. 7 is evident across all datasets and consistent among the individual composites. As storm intensity increases, spectral power increases across all scales. Spectra for all Saffir-Simpson categories exhibit a $k^{-5 / 3}$ spectral slope at high wavenumbers (wavelengths less than roughly $20 \mathrm{~km}$ ) and gradually steeper slopes as the curves progress toward low wavenumbers. The scale at which steepening first occurs appears to be smaller for stronger storms. Figure 10 depicts this downscale shift for the joint composite, showing that the cat-5 power spectrum steepens between 11 and $18 \mathrm{~km}$, cat 4 between 18 and $28 \mathrm{~km}$, and cat 3 between 28 and $43 \mathrm{~km}$ (cat 3 may arguably steepen between 18 and $28 \mathrm{~km}$ also). Initial steepening for cat 2 and cat 1 is somewhat ambiguous but is around $28-43 \mathrm{~km}$.

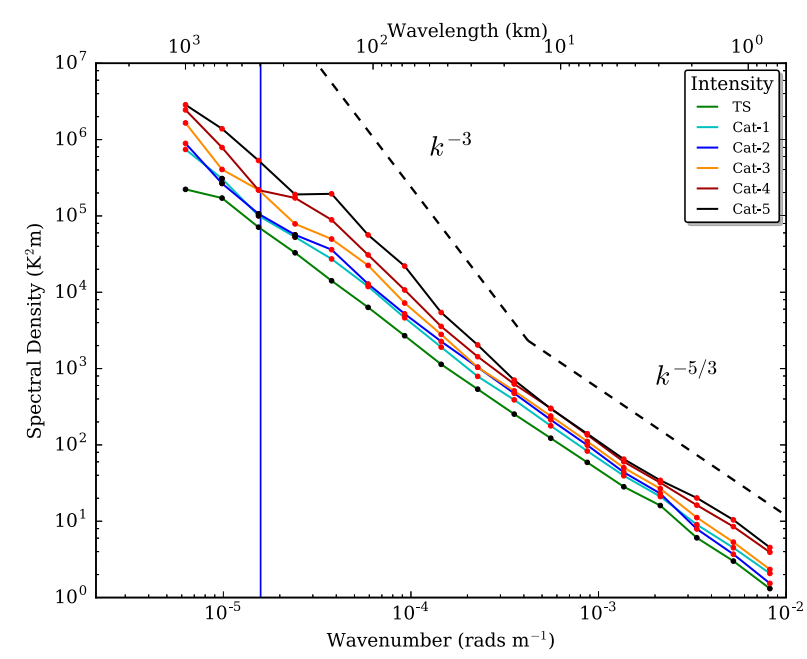

FIG. 8. Spectral composites of all three datasets. Flight legs are truncated at RMW. 


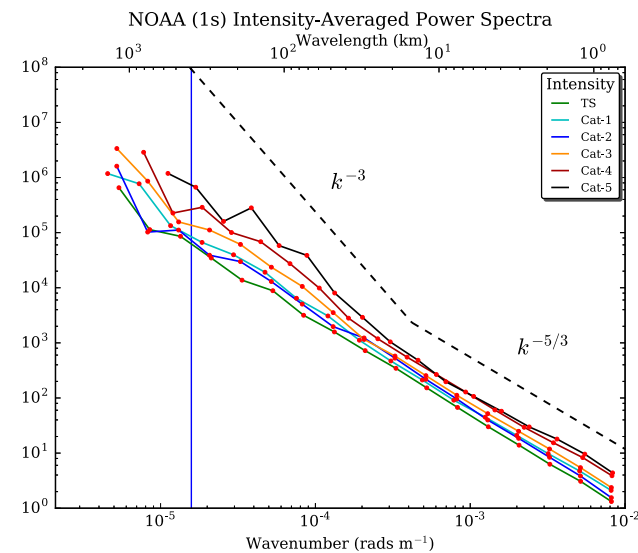

(a)

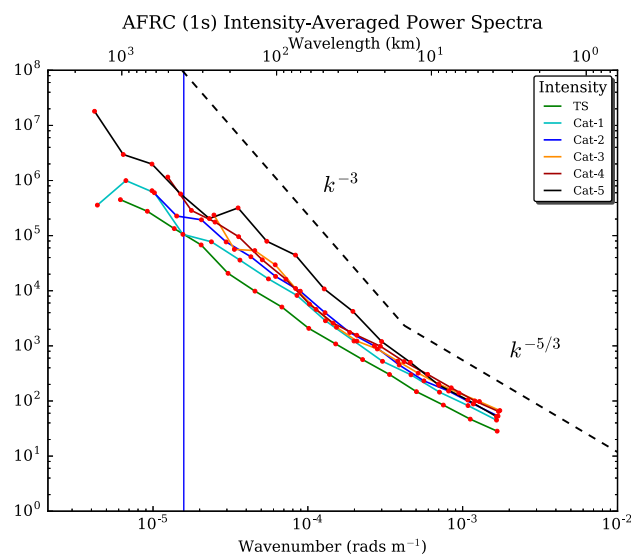

(b)

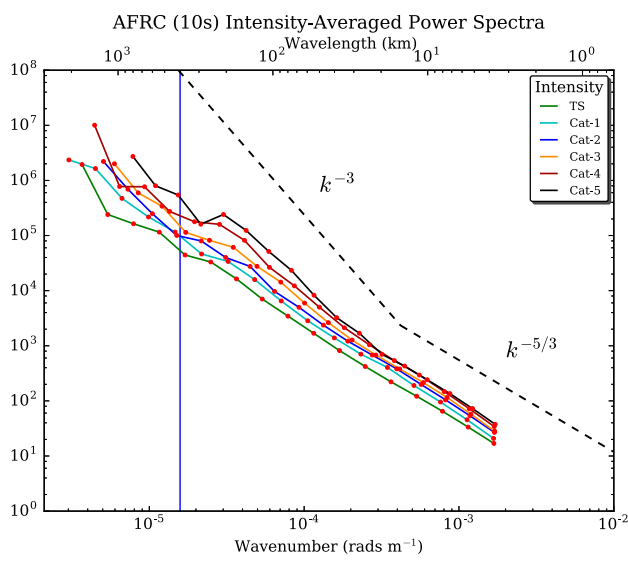

(c)

FIG. 9. Power spectra composites for the (a) NOAA, (b) AFRC 1-s, and (c) AFRC 10-s datasets color coded by category. The blue vertical line serves as a reference for a wavelength of $400 \mathrm{~km}$. Bin means (red circles) are located at the center of each bin along the $x$ axis. In order of sample size, the AFRC 10-s composite is composed of the most missions (749), followed by AFRC 1 s (302) and NOAA (229).

The TS power spectrum shows slightly elevated slopes between 43 and $166 \mathrm{~km}$ but is close to a $k^{-5 / 3}$ slope across all scales. The region of greatest power is generally found between 50 and $200 \mathrm{~km}$ regardless of storm intensity. Although a $k^{-5 / 3}$ spectral slope exists at wavelengths less than roughly $20 \mathrm{~km}$, the cat- 4 and -5 spectra show elevated amplitudes at wavelengths less than $5 \mathrm{~km}$. Figure 9a captures this increased power, which could be connected to enhanced convective activity in stronger storms.

A summary of statistics regarding the flights and their spectra is given in Table 1. The steepest slope of $k^{-3.1}$ is found in the 43-68-km wavelength bin of cat-5 storms; cat -5 storms also have the steepest mean slope, defined as the average over $4.6-166 \mathrm{~km}$, of $k^{-2.2}$. Mean slope and maximum slope generally increase with increasing storm strength. A dip in the power spectra can be seen around $200 \mathrm{~km}$ across all three datasets in addition to the joint composite. It is more pronounced for stronger storms and nonexistent for the TS curve. We hypothesize that this dip represents a return to the "background" KE spectrum at scales not influenced by hurricanes. This explains the flat or occasionally positive slopes (see Fig. 10) at low wavenumbers. At the lowest wavenumbers, the small number of samples used to produce these averages contributes to the slope variability. This is particularly apparent in the individual dataset composites (Fig. 9). As mentioned earlier, there are just 96 flight legs greater than $1000 \mathrm{~km}$ in the joint composite. With these legs spread across six intensity categories and three datasets, the large slope variability at the low wavenumbers - particularly for the individual compositesis not surprising. Slope variability also tends to increase with storm intensity since, naturally, the Hurricane Hunters have intercepted fewer cat-5 hurricanes than cat-1 hurricanes. For example, the AFRC1-s composite only has 22 flight legs contributing to the cat-5 power spectrum. Slope variability decreases at all scales for the joint composite because of the 


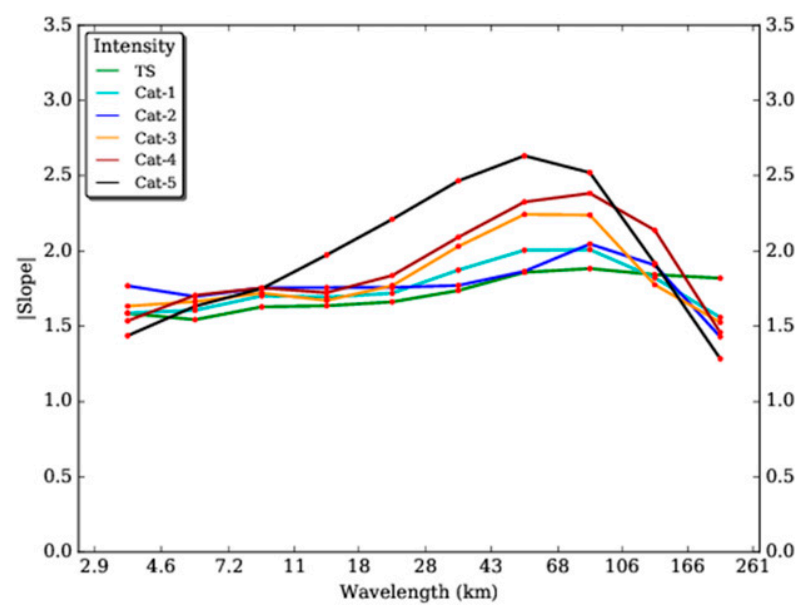

FIG. 10. The points represent the bin-to-bin slopes in Fig. 8, where the $x$ axis is now expressed in wavelength. From left to right, slopes gradually increase until reaching their respective maxima (generally between 43 and $106 \mathrm{~km}$ ) then decrease as the power spectrum returns to "background" levels, and finally, the slopes diverge at scales where few flight legs exist. Slopes at the smallest scales exhibit noticeable variability - this is where the NOAA data are merged with the AFRC data.

greater number of flight legs. Though we have established that the cat-4 and -5 spectra have increased power below $5 \mathrm{~km}$, the apparent tightening of mean values in the joint composite at $3.1 \mathrm{~km}$ is an artifact of the NOAA dataset being spliced with the AFRC data as established in section $3 \mathrm{c}$.

The TS curve in the joint composite (Fig. 8) has mean values designated by black-filled circles compared to the usual red-filled circles. It serves as a reference curve for testing the difference of two means using a two-tailed $z$ test. Mean values displayed in red for the joint composite are found to be different than the vertically aligned reference mean (black-filled circle) at the $99 \%$ confidence level. All mean values pass this test with the exception of the cat- 1 and -2 mean values at 408 and $638 \mathrm{~km}$. When calculating bin averages, values assigned to a given bin are assumed to be independent from one another. This is not strictly true considering NOAA and AFRC crews may simultaneously sample the same storm, but whenever this does happen, crews make a point to sample different regions so as to avoid redundant information. On the whole, flight legs are well diversified both spatially and temporally.

\section{b. Discussion}

Smith and Waleffe (1999) conducted a host of energy spectra experiments investigating the impact of rotation on spectral slope. Lindborg (2005) expanded on their work, showing that below a critical Rossby number (i.e., in the limit of strong rotation), an inhibition of forward energy cascade occurs. This leads to an accumulation of energy at scales greater than the forcing scale, steepening the slope.
Given that these studies are based upon quasigeostrophic (QG) dynamics as opposed to the gradient flow of a hurricane, they are not necessarily applicable. Nevertheless, they may offer some insight into the steeper spectra observed for stronger storms.

In terms of predictability, steeper spectral slopes suggest that stronger storms may be more predictable than weaker storms. Addressing this issue is beyond the scope of this paper, but we note that a modeling review conducted by Bhatia and Nolan (2013) supports this idea, having established that skill scores for the GFDL and National Hurricane Center official (OFCL) hurricane forecasts are positively correlated with model 0-h intensity.

Another issue confounding interpretation of the spectra results presented here is the anisotropic and inhomogeneous aspects of hurricane structure. This motivated our null-hypothesis spectrum discussed in section 3c, which provides an alternative possibility to turbulence for explaining the spectra we observe. A sample of potential temperature spectra derived from the NOAA dataset provides additional insight (Fig. 11). In contrast to Nastrom and Gage (1985), where spectral slopes for wind and potential temperature are in good agreement across all scales, here, we find significant differences. Specifically, the potential temperature spectra follow an approximately $k^{-5 / 3}$ spectral slope across all scales and storm intensity, with only the cat-5 spectrum deviating from the lower-intensity spectra below a wavelength of $30 \mathrm{~km}-$ a deviation likely caused by an outlier disproportionately impacting the small cat-5 potential temperature sample. Clearly, much further research is necessary in order to differentiate the effects of vortex structure and turbulence on hurricane spectral slope.

\section{Summary and conclusions}

We have produced power spectra composites for hurricanes as a function of storm intensity using data from NOAA and AFRC Hurricane Hunter missions. Over 1280 missions (7244 flight legs) were analyzed. To the best of our knowledge, this is the first extensive investigation of hurricane power spectra using in situ aircraft data. As hurricane wind speeds increase, spectral slope steepens over wavelengths of about $10-200 \mathrm{~km}$, with shallower slopes at smaller and larger scales. Shallower slopes at larger scales are especially apparent for the cat $-3,-4$, and -5 composites. Additionally, we find that the scale at which slope begins to steepen moves downscale as storm category increases. The cat-5 composite achieves the steepest slope of -3.1 , while the TS composite only reaches a slope of -1.9 .

To better examine any predictability ramifications of hurricane power spectra, future work could begin with producing composites for other variables such as temperature and moisture. Spectra for these variables should provide 


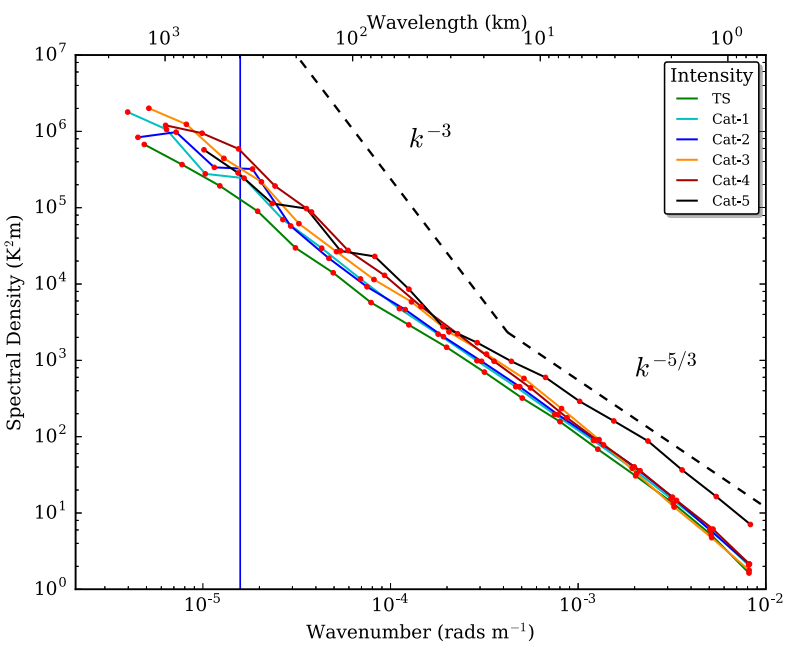

FIG. 11. NOAA potential temperature composites.

insight as to whether the spectra inform on hurricane structure or turbulence. Another useful approach could involve the investigation of flight paths with a nearly constant radius from the storm center. Since storm structure varies most radially, data from these flights should have less influence from this aspect of storm structure. Such a study may benefit from comparison with results for numerical simulations because of the rarity of missions with circular flight paths. Other possible investigations include stratifying hurricanes by RMW, examining the effects of secondary eyewalls, and determining the power spectra of horizontal divergence and vertical vorticity as in Lindborg (2007).

Acknowledgments. This study was supported by the NSF Graduate Research Fellowship Program (2015199752) and the NOAA HFIP (NA14NWS4680031). This paper forms a portion of the first author's master's thesis at the University of Washington. We thank Neal Dorst, Jack Parrish, Ashley Lundry, Sonia Otero, and Sim Aberson for information regarding data formatting and flight procedures. Special thanks to the crews of the AFRC and the NOAA Hurricane Hunters, without whom this research would not have been possible.

\section{APPENDIX}

\section{Standard Operating Procedures and Data Quality}

\section{a. Aircrew standard operating procedures}

Each storm intercept is complemented by a corresponding mission summary and accompanying radar data. Flight tracks generally resemble a "figure 4" over investigated storms, with intercepting flight legs oriented along intercardinal $\left(45^{\circ}, 135^{\circ}\right.$, $225^{\circ}, 315^{\circ}$ ) directions. Once crews have committed to an intercept, every effort is made to maintain a straight flight path and consistent speed through the RMW (Federal Coordinator for Meteorological Services and Supporting Research 2017). Flights are executed along a 925-, 850-, or $700-\mathrm{hPa}$ pressure surface depending on whether the target storm is a tropical invest, tropical depression/storm, or hurricane (Federal Coordinator for Meteorological Services and Supporting Research 2017). Flight leg length varies based on storm diameter and customer priorities, but $96 \%$ of legs used in this study are $300 \mathrm{~km}$ or less. Recorded variables and aircraft type have fluctuated over the half century that hurricane-hunting operations have transpired, but they remain fairly consistent from decade to decade. Official mission data date back to Hurricane Donna in 1960, but the U.S. Navy and the U.S. Weather Bureau conducted flights even earlier. More detail about the NOAA and AFRC Hurricane Hunters standard operating procedures can be found in the publicly available national hurricane operations plan.

\section{b. NOAA and AFRC data quality}

As a form of quality control, software managing the AFRC 10-s data requires a minimum of 7 data points to be present in a given 10-s period for a 10-s average to be recorded. If fewer than 7 data points are available to contribute to the 10-s average, that line is coded as missing data. In the AFRC 1-s dataset, 10 data points must be present in order for a 30-s average to be valid. NOAA data were either present or missing for a given second with no specified quality-control measures. Further detail about AFRC data quality can be found in the Weather Bird software logic and algorithms manual (available through the 53WRS; 53rd Weather Reconnaissance Squadron 2016).

Missing data were rare: roughly 1 in 190 observations for the NOAA and AFRC 1-s data and 1 in 355 observations for the AFRC 10-s data. Spectral analysis was performed on all flight legs regardless of missing data and then only performed on flight legs with no missing data. Results varied negligibly, so all available flight legs were utilized. Outliers caused by instrument error were only present in flight data from Hurricane Allen (1980) and Hurricane Gilbert (1988). These outliers were removed before analysis.

\section{REFERENCES}

53rd Weather Reconnaissance Squadron, 2016: Weather Bird software logic and algorithms. U.S. Air Force.

Balsley, B. B., and D. A. Carter, 1982: The spectrum of atmospheric velocity fluctuations at $8 \mathrm{~km}$ and $86 \mathrm{~km}$. Geophys. Res. Lett., 9, 465-468, https://doi.org/10.1029/GL009i004p00465.

Bhatia, K. T., and D. S. Nolan, 2013: Relating the skill of tropical cyclone intensity forecasts to the synoptic environment. Wea. Forecasting, 28, 961-980, https://doi.org/10.1175/WAF-D-12-00110.1.

Chen, T.-C., and A. Wiin-Nielsen, 1978: On nonlinear cascades of atmospheric energy and enstrophy in a two-dimensional 
spectral index. Tellus, 30, 313-322, https://doi.org/10.3402/ tellusa.v30i4.10346.

Durran, D., J. A. Weyn, and M. Q. Menchaca, 2017: Practical considerations for computing dimensional spectra from gridded data. Mon. Wea. Rev., 145, 3901-3910, https://doi.org/10.1175/ MWR-D-17-0056.1.

Federal Coordinator for Meteorological Services and Supporting Research, 2017: National hurricane operations plan. NOAA Tech. Rep. FCM-P12-2016, 167 pp., http://www.ofcm.gov/ publications/nhop/FCM-P12-2017.pdf.

Hsu, S., and Z. Yan, 1998: A note on the radius of maximum wind for hurricanes. J. Coastal Res., 14, 667-668.

Khain, A., B. Lynn, and J. Dudhia, 2010: Aerosol effects on intensity of landfalling hurricanes as seen from simulations with the WRF Model with spectral bin microphysics. J. Atmos. Sci., 67, 365-384, https://doi.org/10.1175/2009JAS3210.1.

Lilly, D. K., and E. L. Petersen, 1983: Aircraft measurements of atmospheric kinetic energy spectra. Tellus, 35A, 379-382, https://doi.org/10.3402/tellusa.v35i5.11448.

Lindborg, E., 2005: The effect of rotation on the mesoscale energy cascade in the free atmosphere. Geophys. Res., 32, L01809, https://doi.org/10.1029/2004gl021319.

, 2007: Horizontal wavenumber spectra of vertical vorticity and horizontal divergence in the upper troposphere and lower stratosphere. J. Atmos. Sci., 64, 1017-1025, https://doi.org/ 10.1175/JAS3864.1.

Lorenz, E. N., 1969: The predictability of a flow which possesses many scales of motion. Tellus, 21, 289-307, https://doi.org/ 10.3402/tellusa.v21i3.10086.
Nastrom, G. D., and K. S. Gage, 1985: A climatology of atmospheric wavenumber spectra of wind and temperature observed by commercial aircraft. J. Atmos. Sci., 42, 950-960, https://doi.org/ 10.1175/1520-0469(1985)042<0950:ACOAWS > 2.0.CO;2.

Rogers, R., P. Reasor, and S. Lorsolo, 2013: Airborne Doppler observations of the inner-core structural differences between intensifying and steady-state tropical cyclones. Mon. Wea. Rev., 141, 2970-2991, https://doi.org/10.1175/MWR-D-12-00357.1.

Rosenfeld, D., A. Khain, B. Lynn, and W. L. Woodley, 2007: Simulation of hurricane response to suppression of warm rain by sub-micron aerosols. Atmos. Chem. Phys., 7, 3411-3424, https://doi.org/10.5194/acp-7-3411-2007.

— M. Clavner, and R. Nirel, 2011: Pollution and dust aerosols modulating tropical cyclones intensities. Atmos. Res., 102, 6676, https://doi.org/10.1016/j.atmosres.2011.06.006.

Rotunno, R., and C. Snyder, 2008: A generalization of Lorenz's model for the predictability of flows with many scales of motion. J. Atmos. Sci., 65, 1063-1076, https://doi.org/10.1175/ 2007JAS2449.1.

Skamarock, W. C., 2004: Evaluating mesoscale NWP models using kinetic energy spectra. Mon. Wea. Rev., 132, 3019-3032, https://doi.org/10.1175/MWR2830.1.

Smith, L. M., and F. Waleffe, 1999: Transfer of energy to twodimensional large scales in forced, rotating three-dimensional turbulence. Phys. Fluids, 11, 1608-1622, https://doi.org/10.1063/ 1.870022.

Vinnichenko, N. K., 1970: The kinetic energy spectrum in the free atmosphere-1 second to 5 years. Tellus, 22, 158-166, https:// doi.org/10.3402/tellusa.v22i2.10210. 